\title{
5 Was ist ein QM-Handbuch?
}

Julia Bellabarba

Die Erstellung eines QM Handbuchs ist sinnvoll, unabhängig von der Systematik, die genutzt wird.

- Was bringt das QM Handbuch? Es stellt Transparenz und Verbindlichkeit nach außen und nach innen dar.

- Was wird dokumentiert? Die wichtigsten Unternehmensziele, Standards, Prozessabläufe, Arbeitsanweisungen, Dokumente, Strukturen, Verantwortlichkeiten der Praxis.

- Transparenz nach außen: für wen? Kostenträger, Gericht, KV, Kooperationspartner, Patienten

- Transparenz nach innen: für wen? Alle (insbesondere neue) Mitarbeiter/-innen

- Transparenz nach innen in der Psychotherapiepraxis: für wen? Psychotherapeuten sind auch nur Menschen. Insofern dienen in der psychotherapeutischen Einzelpraxis entsprechende Ablaufbeschreibungen und Dokumente, insbesondere Checklisten, die kontinuierlich weiterentwickelt werden, der eigenen Sicherheit und Verbesserung („Ich reflektiere, was ich tue, und prüfe, obdas, wasich tue, wirksam ist“).

Handbücher enthalten also die Dokumentation gelebten Qualitätsmanagements und schaffen Verbindlichkeit.

Die eingangs erwähnte Richtlinie des Gemeinsamen Bundesausschusses fordert eine schriftliche Dokumentation, also das Führen und Aktualisieren eines QM Handbuchs, explizit. Eine Praxis muss also, um den formalrechtlichen Vorgaben gerecht zu werden, ein QM-Handbuch anlegen, egal nach welcher Systematik, egal ob in Papierform oder digital. Darüber hinaus gibt das Handbuch möglichen Vertragspartnern oder externen Visitoren im Rahmen 
einer Zertifizierung die Möglichkeit, sich über die Qualität der Leistungen einer Praxis zu informieren. Die QM-Kommissionen der KV prüfen jährlich 2,5\% der Praxen stichprobenartig auf Konformität mit der Richtlinie („Aufforderung zum schriftlichen Nachweis“). Diese Prüfung wird in Form einer Selbsteinschätzung erfolgen, QM-Handbücher werden in diesem Zusammenhang vorerst nicht abverlangt.

Ein Handbuch ist nicht mehr und nicht weniger als beschriebenes Papier: Gelebtes Qualitätsmanagement ersetzt es nicht. Anders ausgedrückt: Qualitätsmanagement ist das, was man macht, das Handbuch ist das, was im Schrank steht. Der Wert eines Handbuchs ist umso größer, desto ökonomischer bei der Dokumentation vorgegangen wird: so wenig wie möglich, so viel wie nötig, ist auch hier die Devise. 